\title{
Predicting outcomes in patients with perforated gastroduodenal ulcers: artificial neural network modelling indicates a highly complex disease
}

\author{
K. Søreide $\cdot$ K. Thorsen $\cdot$ J. A. Søreide
}

Received: 11 April 2014 / Accepted: 26 May 2014/Published online: 14 June 2014

(C) The Author(s) 2014. This article is published with open access at Springerlink.com

\begin{abstract}
Purpose Mortality prediction models for patients with perforated peptic ulcer (PPU) have not yielded consistent or highly accurate results. Given the complex nature of this disease, which has many non-linear associations with outcomes, we explored artificial neural networks (ANNs) to predict the complex interactions between the risk factors of PPU and death among patients with this condition.

Methods ANN modelling using a standard feed-forward, back-propagation neural network with three layers (i.e., an input layer, a hidden layer and an output layer) was used to predict the 30-day mortality of consecutive patients from a population-based cohort undergoing surgery for PPU. A receiver-operating characteristic (ROC) analysis was used to assess model accuracy.

Results Of the 172 patients, 168 had their data included in the model; the data of $117(70 \%)$ were used for the training set, and the data of 51 (39\%) were used for the test set. The accuracy, as evaluated by area under the ROC curve (AUC), was best for an inclusive, multifactorial ANN model (AUC 0.90, $95 \%$ CIs 0.85-0.95; $p<0.001$ ). This model outperformed standard predictive scores, including Boey and PULP. The importance of each variable decreased as the number of factors included in the ANN model increased.

Conclusions The prediction of death was most accurate when using an ANN model with several univariate
\end{abstract}

K. Søreide $(\bowtie) \cdot$ K. Thorsen · J. A. Søreide

Department of Gastrointestinal Surgery, Stavanger University

Hospital, P.O. Box 8100, 4068 Stavanger, Norway

e-mail: ksoreide@mac.com

K. Søreide · K. Thorsen · J. A. Søreide

Department of Clinical Medicine, University of Bergen, Bergen, Norway influences on the outcome. This finding demonstrates that PPU is a highly complex disease for which clinical prognoses are likely difficult. The incorporation of computerised learning systems might enhance clinical judgments to improve decision making and outcome prediction.

Keywords Peptic ulcer perforation - Gastroduodenal ulcers · Mortality · Prediction · Prognosis · Outcome assessment $\cdot$ Computer simulation

\section{Introduction}

Perforated peptic ulcers (PPUs) are the leading cause of surgery-related death worldwide [1]. Although the incidence of peptic ulcer complications due to bleeding has decreased [2], the incidence of perforations has remained stable over the past few decades despite surgical and medical advancements. PPU is a severe complication of peptic ulcer disease with a reported mortality of approximately 10-20\%, even in modern surgical series [3-7].

Mortality prediction is of importance, but previous models have yielded inconsistent results $[8,9]$. Further, the Boey score [10] as one of the most frequently used scores was created on patient series during the 1980s, which may explain why results vary across studies [9]. In fact, we recently demonstrated that no predictive models has superior accuracy [11]; at best, only 4 out of 5 patients are correctly classified by any particular model.

Notably, although prediction was improved by a previous model that combined six pre-operatively obtainable variables [11], the prognostic value of any single factor was limited. This limitation might be explained by the fact that biological systems have relationships between the variables that are complex, multidimensional and non-linear. 
The increasing availability of electronic medical information that can be collected and used for pattern recognition has created new opportunities to improve diagnoses and predictions of disease outcomes [12-15]. Computers can gather and process thousands of variables as well as learn to recognise patterns by simulated "trial-and-error" processing - often referred to as "artificial intelligence". One such type of artificial intelligence is the artificial neural network (ANN). ANNs are information-processing paradigms inspired by the analytical processes of the human brain. Emerging data have demonstrated the superiority of ANN modelling with regard to several benign or malignant gastrointestinal disorders [16-19]; however, ANNs have never been applied to predict PPU outcomes. Thus, our objective was to explore the ability of an ANNto improve survival prediction.

\section{Materials and methods}

The study was approved as a quality control assurance project according to the Regional Ethics Committee (REK Vest \# 2011/713). The study complied with the Strengthening the Reporting of Observational Studies in Epidemiology (STROBE) statement, where applicable [20].

The study cohort has been described in detail elsewhere [11]. A population-based consecutive series of 172 patients diagnosed and operated on for perforated gastroduodenal ulcer between January 2001 and December 2010 were included in the current study. Patients with perforations caused by malignant disease (i.e., gastric cancer) and patients who did not undergo surgery were excluded from the current study. The primary endpoint of this study was the 30-day mortality after surgery for PPU.

The clinical and laboratory variables have been defined previously [11]. Optimal cut-off values were established using ROC analysis for dichotomising continuous variables, as described elsewhere [21]. Both the Boey score [22] and the PULP score [23] have been described elsewhere.

\section{Predicted probabilities from regression analyses}

A multivariate regression analysis was performed as previously described [11] to evaluate the current PPU scoring systems, including Boey and PULP. For the regression models, the saved probabilities for each patient in the model (either Boey or PULP) were used for comparison with the output values generated for ANN modelling in the current study.

In the previous multivariate regression model that used the same material [11], mortality was best predicted based on a combination of negative prognostic factors: increasing age, the presence of an active cancer, a delay from admission to surgery $>24 \mathrm{~h}$, hypoalbuminaemia, hyperbilirubinaemia and increasing creatinine values. The predictive probabilities for the multivariate model had an AUC of 0.89 .

\section{Statistical analyses}

The data were analysed using the Statistical Package for Social Sciences (IBM SPSS v. 21, Inc. for Mac).

The ANN model used in the current study was a multilayer perceptron (MLP) network conducted as a standard feed-forward, back-propagation neural network with three layers: an input layer, a hidden layer and an output layer. The MLP network is a tool for designing special classes of layered feed-forward networks. The input layer consists of source nodes, and its output layer consists of neurons; these layers connect the network to the outside world. In addition to these layers, the MLP usually has one or more layers of neurons referred to as hidden neurons because they are not directly accessible. The hidden neurons extract important features contained in the input data.

The patients were randomly divided into a training/ cross-validation group $(70 \%)$ and an internal validation group $(30 \%)$. The training/cross-validation group was used to train the network.

We initially constructed the present MLP network with six input neurons derived from the previous logistic regression model, which included only objective and reproducible laboratory variables (i.e., age, surgical delay, the presence of active cancer and blood laboratory values for serum albumin, bilirubin and creatinine).

\section{Definitions of the ANN models}

The experimental design is visualised in Fig. 1. The first model (hereafter referred to as model \#1) was created using the same input variables as previously established in uniand multivariate regression analyses [11], with dichotomised variables for continuous data based on optimal cutoff values in the ROC analysis (e.g., to indicate hypoalbuminaemia, hyperbilirubinaemia and increased creatinine values; Table 1). In the second model (model \#2), the same variables were included but used as non-dichotomised, continuous values to allow the model to adjust the role of each variable throughout the spectrum of values.

Finally, a third model (model \#3) was created to explore the enhanced capabilities and unknown interactions of variables. This model included all of the potential factors that might be associated with mortality, including gender, the presence of shock at admission, sepsis and mode of surgery.

To compare these three models, the output predictive value for each ANN was compared using ROC analysis and 
Fig. 1 Flow-chart of experiment for each model. Asterisk regression based on variables selected in multivariable analyses from Thorsen et al. [11]

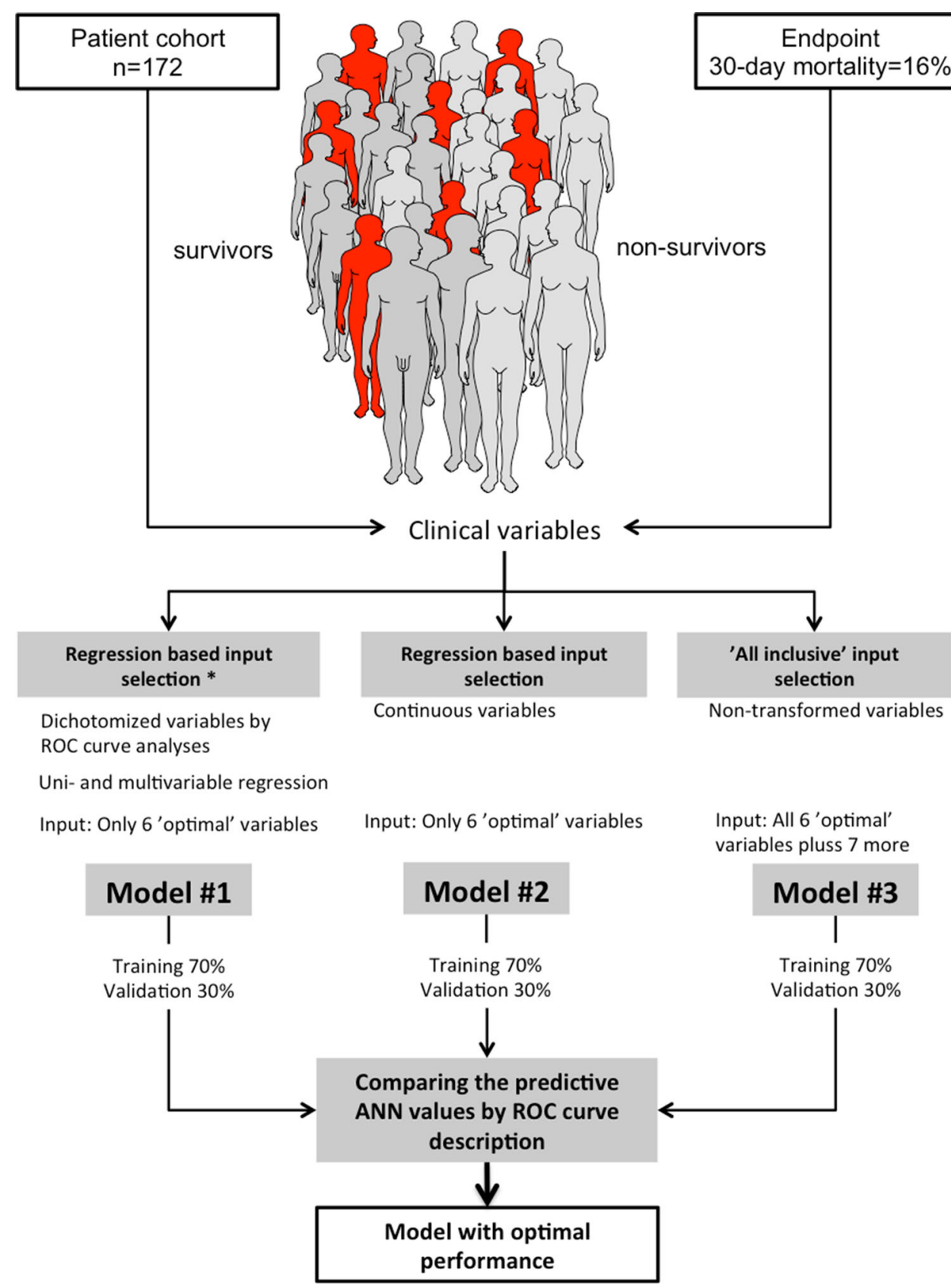

$95 \%$ confidence intervals. All $p$ values $<0.050$ were considered significant.

\section{Results}

A total of 172 patients were included; 28 deaths occurred within 30 days of surgery (16\%). The baseline data are presented in Table 2. The networks created for ANN models \#1 and \#2 are shown in Fig. 1. The six input nodes resulted in different hidden layers for outcome predictions when either dichotomised (Fig. 2a) or continuous (Fig. 2b) variables were imputed. Notably, the accuracy of the model was reduced by the latter approach (Table 3 ).

ANN model \#1 had an accuracy that was somewhat lower than that of a previously reported regression model. The AUC of the ANN was 0.84, whereas the AUC of the logistic regression model was 0.89 using the same variables [11]. The accuracy of the model decreased (model \#2) when the variables were input as continuous measures (Table 3). Increasing the available information in the model by liberally including numerous variables (ANN model \#3) with an unknown relationship to the outcome improved the accuracy of the model to AUC 0.90 (Table 3; 
Table 1 Variables included in the neural network modelling
For comparison, the variables included in the Boey and PULP scores are shown

\begin{tabular}{|c|c|c|c|c|c|}
\hline Factors (units) & ANN mod. \#1 & ANN mod. \#2 & ANN mod. \#3 & Boey & PULP \\
\hline Gender (M/F) & & & $\checkmark$ & & \\
\hline Age (years) & $\boldsymbol{V}$ & $\checkmark$ & $\checkmark$ & & $\boldsymbol{\nu}$ \\
\hline Location of ulcer (duodenal/gastric) & & & $\checkmark$ & & \\
\hline Diagnostic delay $(\mathrm{h})$ & & & & & $\checkmark$ \\
\hline Delay before surgery $(\mathrm{h})$ & $\checkmark$ & $\checkmark$ & $\checkmark$ & $\checkmark$ & \\
\hline Type of surgical repair (lap/open) & & & $\checkmark$ & & \\
\hline Comorbidity (any) & & & & $\checkmark$ & \\
\hline ASA fitness score (I-V) & & & $\checkmark$ & & $\boldsymbol{V}$ \\
\hline Active cancer disease $(y / n)$ & $\boldsymbol{v}$ & $\boldsymbol{v}$ & $\checkmark$ & & $\boldsymbol{V}$ \\
\hline Liver cirrhosis $(\mathrm{y} / \mathrm{n})$ & & & & & $\boldsymbol{V}$ \\
\hline Steroid use $(\mathrm{y} / \mathrm{n})$ & & & & & $\boldsymbol{v}$ \\
\hline Albumin $(\mathrm{g} / \mathrm{L})$ & $\checkmark$ & $\checkmark$ & $\checkmark$ & & \\
\hline Bilirubin $(\mu \mathrm{mol} / \mathrm{L})$ & $\checkmark$ & $\boldsymbol{V}$ & $\checkmark$ & & \\
\hline Creatinine $(\mu \mathrm{mol} / \mathrm{L})$ & $\checkmark$ & $\boldsymbol{V}$ & $\checkmark$ & & $\boldsymbol{\nu}$ \\
\hline Leucocytes $\left(10^{9} / \mathrm{L}\right)$ & & & $\checkmark$ & & \\
\hline C-reactive protein $(\mathrm{mg} / \mathrm{L})$ & & & $\checkmark$ & & \\
\hline Sepsis on admission $(\mathrm{y} / \mathrm{n})$ & & & $\checkmark$ & & \\
\hline Shock on admission $(\mathrm{y} / \mathrm{n})$ & & & $v$ & $\boldsymbol{\nu}$ & $\boldsymbol{\nu}$ \\
\hline
\end{tabular}

Fig. 3). In clinical terms, this result means that the model should accurately predict death in 9 out of 10 patients undergoing an operation for PPU.

The constructed graphs for each model in the network analysis suggest that accuracy increased when more variables were added to the model (Fig. 3). When including additional variables such as the presence of comorbidities (e.g., cardiovascular, pulmonary or autoimmune diseases), the accuracy of the model decreased (data not shown), which indicates that additional predictive information was not available.

Furthermore, as the number of variables in the models increased, the predictive value of each variable decreased considerably (Fig. 4a-c). In fact, the incremental input from each variable was small (Fig. 4c), although the model's prediction accuracy increased (Fig. 3). In addition, the relative contribution of each variable changed considerably (Fig. 4a-c), as expressed by the variable importance.

The weighted importance of the factors changed; a strong emphasis was placed on CRP, creatinine and bilirubin; however, the other factors (including age, shock, active cancer or other risk factors) exerted virtually no effect.

ANN model \#3 was more accurate than a previously reported multivariate regression model based on increasing age, the presence of active cancer, an admission delay for surgery of $>24 \mathrm{~h}$, hypoalbuminaemia, hyperbilirubinaemia and increased creatinine values (model AUC of 0.89) [11]. However, the increase in accuracy was not significant
Table 2 Baseline characteristics of the patients with PPU

\begin{tabular}{lll}
\hline Characteristics & $\begin{array}{l}\text { Total } \\
(n=172)\end{array}$ & $\begin{array}{l}\text { Deaths at 30 } \\
\text { days }(n=28)\end{array}$ \\
\hline Gender, F:M & $89: 83$ & $17: 11$ \\
Age, median years (range) & $68(18-100)$ & $80(56-95)$ \\
Ulcer in prior history $(n, \%)$ & $26(15 \%)$ & $5(18 \%)$ \\
Location of ulcer, duodenal:gastric & $60: 112$ & $14: 14$ \\
Delay to surgery, median hours & 6.2 & 10.0 \\
(range) & $(0.5-116.2)$ & $(1.1-40.6)$ \\
Laparoscopic repair & $50(29 \%)$ & $8(29 \%)$ \\
Shock at admission & $37(22 \%)$ & $10(37 \%)$ \\
Sepsis at admission & $70(42 \%)$ & $12(48 \%)$ \\
ASA fitness score $\geq$ III & $73(42 \%)$ & $24(86 \%)$ \\
Active cancer disease & $19(11 \%)$ & $9(32 \%)$ \\
Boey score $\geq 2$ & $56(33 \%)$ & $18(64 \%)$ \\
Steroid use & $16(10 \%)$ & $5(18 \%)$ \\
\hline
\end{tabular}

The data are presented as numbers and (\%) or as medians with ranges

(from an AUC of 0.89-0.90), showing an overlap of the $95 \%$ CIs for both models.

\section{Discussion}

This study demonstrated that the application of ANNs to enhance the outcome predictions regarding patients with PPU slightly improved upon the predictive ability of a previously reported regression model [11]. Furthermore, 


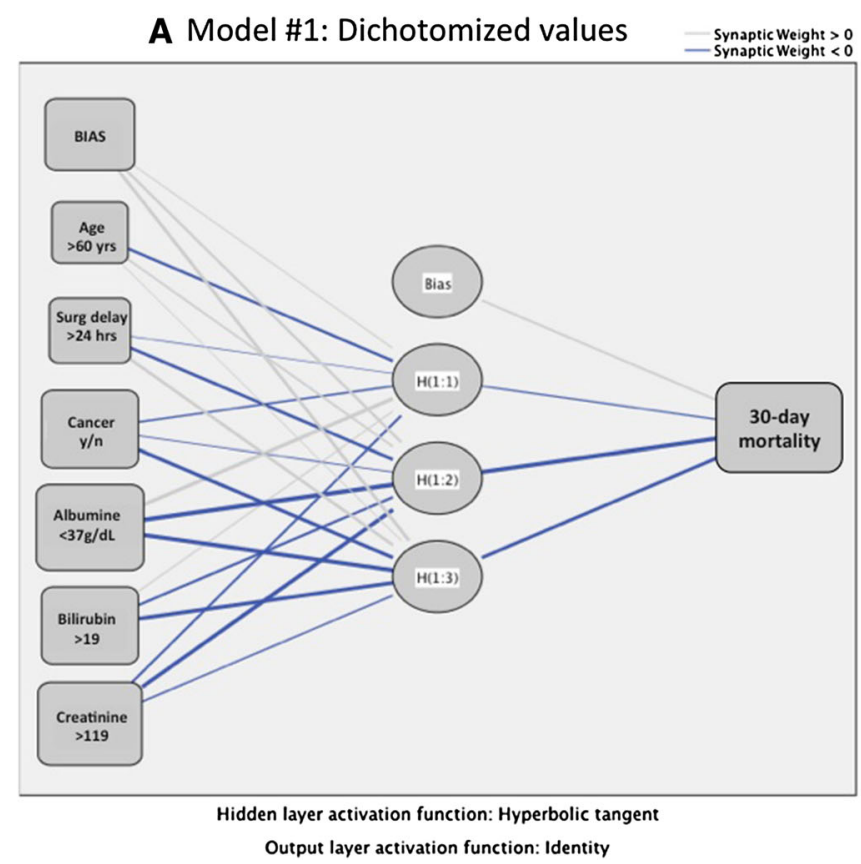

Fig. 2 Two ANN models. Although the same input variable nodes were kept, the network and hidden nodes changed when using continuous data (in model \#2) versus dichotomised values (in model

Table 3 ROC analysis with AUC and $95 \%$ CIs for the different ANN models

\begin{tabular}{llll}
\hline Test result variable(s) & AUC & AUC 95 \% CI & $P$ value \\
\hline Neural network model \#1 & 0.84 & $0.77-0.91$ & $<0.001$ \\
Neural network model \#2 & 0.77 & $0.67-0.87$ & $<0.001$ \\
Neural network model \#3 & 0.90 & $0.85-0.95$ & $<0.001$ \\
\hline
\end{tabular}

the ANNs performed better than the currently proposed Boey and PULP scores for outcome predictions among patients with PPU. Of note, the ANN models suggested a difference in the weighted importance of the included factors that changed based on how the variables were included and combined in the model. Based on the differences in the model inputs and the resulting outputs, outcome predictions of PPU were complex and likely to be influenced by several factors that might have unknown relationships with each other (which cannot be easily demonstrated using standard near-linear, regression models). The cumulative presence of unknown and small relationships might largely explain the difficulty, limited success and moderate accuracy associated with generating predictive models consisting of a few (e.g., 3-5) explanatory variables, given that the actual outcome depends on a much greater variance and (at least partially unknown) interdependence of factors. Drawing an analogy to the clinical setting, the inexperienced physician may be overwhelmed of the number of factors to consider, some with

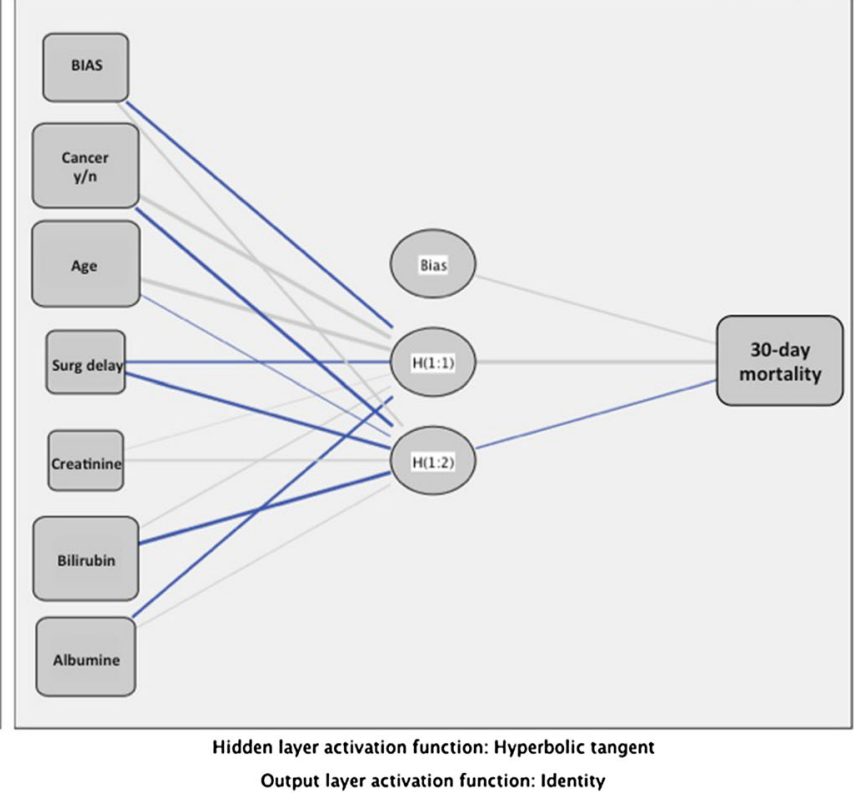

\#1), demonstrating a change in the importance and the relationships between each variable and the outcome

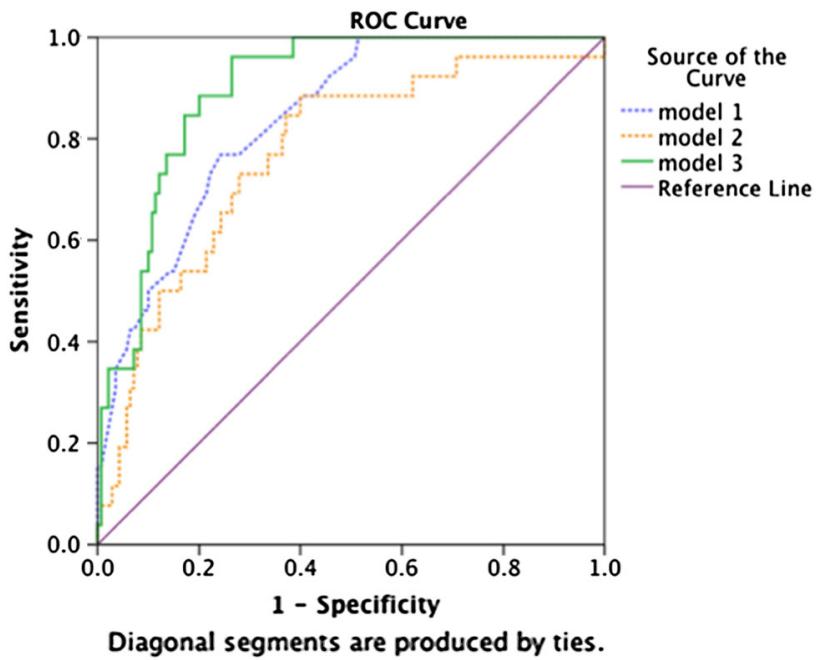

Fig. 3 ROC curve comparing the accuracy of the three ANN models

subtle meaning while others are more predominating, thus preventing the clinician to consider any more than a blunt few for clinical decision making. In reality, many subtle factors may indeed point to a potential dire situation that may easily be missed or go unrecognised by the inexperienced clinician. Situations such as these are where computerised pattern recognition and prediction algorithms can become useful [24]. Although their full potential has yet to be reached [25], developments in technology are rapidly moving toward models that might become available 

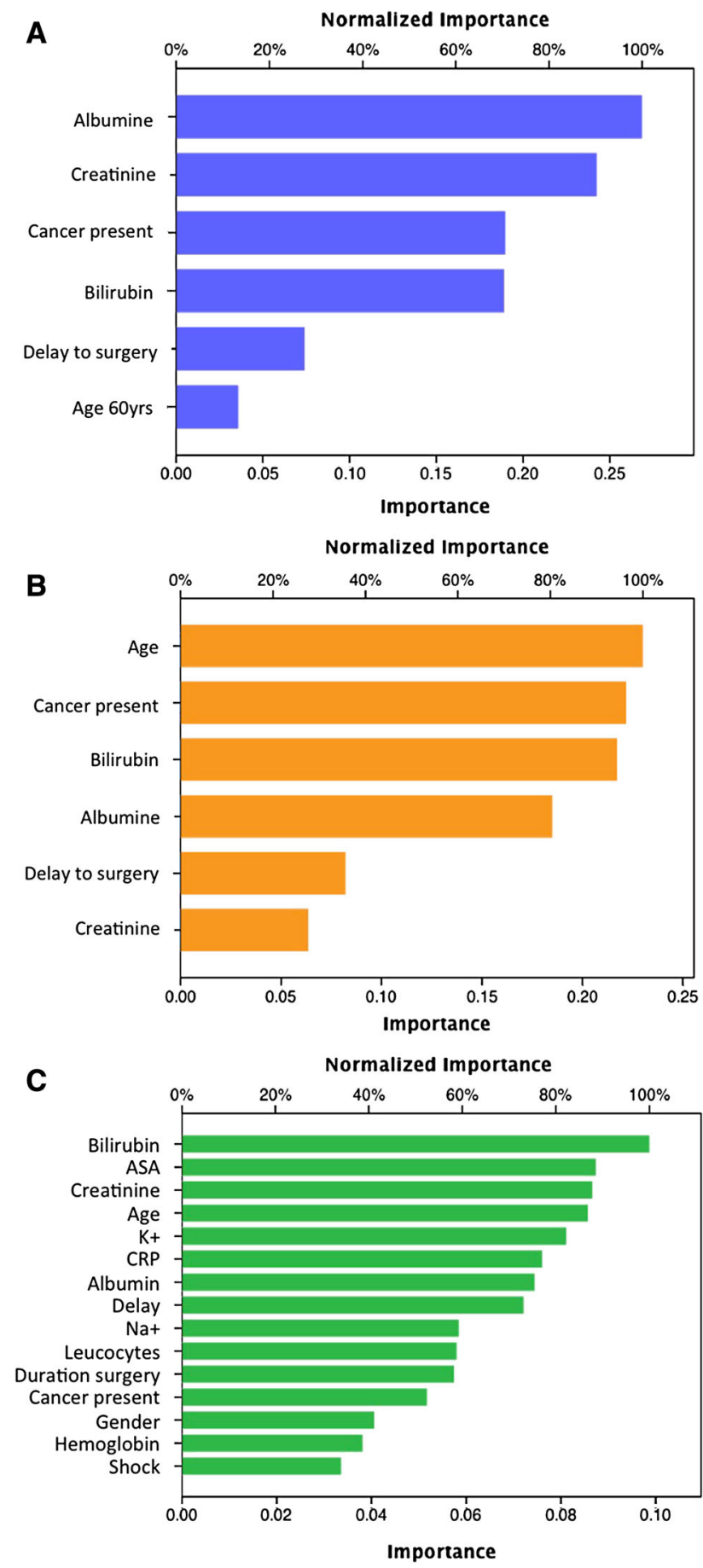

Fig. 4 Weighted importance of included variables for each model. Both variable importance and the relative weight (or contribution) of the predictive models shifted, as indicated on the $x$-axis of each graph $\mathbf{a}$ for model \#1; $\mathbf{b}$ for model \#2 and $\mathbf{c}$ for model \#3

for everyday use. A further example of "innovation technology" might be the rapid and widespread use of information technology such as smart phones and various applications [26] that only a decade ago seemed futuristic and at a developmental stage at best.
ANN-based models might even help expert clinicians. The belief that the brain's short-term memory can simultaneously retain (and therefore optimally use) only 4-7 pieces of information is of importance; attempts to use larger amounts of information at one time can lead to ineffective decision-making. Given this limitation, many clinicians might have difficulty assimilating the many variables that are often encountered in real-life clinical environments. This mismatch between innate human ability (i.e., "human brain processing capacity") and excessive input data (i.e., "information overload") might contribute to unnecessary variations in clinical practice (i.e., "decision-making"), poor compliance with established guidelines and even errors in medical judgment [27]. In fact, certain computer systems are beginning to reveal clinical implications in several areas to improve patient safety and generate complex data analyses [12-14].

Notably, an experienced clinician may outperform the ability of an advanced computer prediction models. However, clinical decision-making is based on the human ability to collect information and process it into clinically predictive patterns. This skill is influenced by knowledge, experience and sources of bias. Knowingly, becoming an "expert" takes thousands of hours of experience-a fortune that clinicians do not have from the start and which may take longer time to require in the current workrestricted environment [28]. The increasing availability of electronic medical information that can be collected and used for pattern recognition has created new opportunities to improve diagnoses and predictions of disease outcomes [12-15]. Computers can gather and process thousands of variables as well as learn to recognise patterns by simulated "trial-and-error" processing, often referred to as "artificial intelligence". In other words, computers are able to make informed decisions. Such technology is already in use in aviation systems, banking technology, industrial robotics and certain areas of medicine [29, 30]. While we do not suggest that computer systems will replace human input, there may be a source for potential improvement in pattern recognition that the inexperienced human brain is incapable of, at least until obtaining thousands hours of experience. It may indeed represent the "gut feeling" of the experienced clinician that recognise a premonition without being able to pin-point the exact determinator for it.

The potential medical applications of ANNs include scenarios in which the relationship between independent variables and clinical outcomes are poorly understood [31, 32]. Because ANNs are capable of self-training with minimal human intervention, many studies of large epidemiology databases have used ANNs in addition to traditional statistical methods to gain additional insight into the relationships among variables. Several previous studies have used ANNs to predict mortality after surgery [16, 33-35]. 
ANNs have also been used to aid in the diagnosis and determination of disease severity $[17,19,36]$. ANNs are particularly suited to solving non-linear problems and analysing complex datasets [32]. As such, ANNs constitute potent alternative computational tools that are able to outperform the diagnostic and prognostic accuracy of classical statistical methods.

Of note, ANNs and other artificial intelligence systems are only as smart as we make them. Thus, there will always be a need for human input of what sort of data, the quality of data and the source of data collection that the ANN may utilise. Accordingly, the danger of "garbage-in, garbage out" may exist. Also, most clinicians (and researchers) may be uncomfortable with the "hidden nodes" which are essentially unavailable and intangible elements of the computer process. Also, if input is obtained on a one-time point basis, it may miss the dynamics of process. However, in real-time models that captures and process variables continuously, this limitation may be overcome. One example may be the continuous monitoring performed in intensive care units, for which considerable data amounts may be difficult to assess for the human brain, but may yield threshold values (i.e., express risk of adverse event or further deterioration) in a learning, artificial intelligence system.

The current strict, population-based, non-selected cohort is a particular strength of this study because it reduces transferral bias or other selection criteria that are found in regions with coverage overlap between hospitals. However, all studies have limitations, and the current study is partially limited by its moderately sized sample and reliance on previously defined predictive variables (e.g., the best cut-off values for dichotomous variables) for building the models. A true, secondary and external validation cohort is lacking. If such a cohort were present, this study would have enhanced generalizability, but no such cohort was available when the project began. However, the cohort was split into training and test sets for the modelling. Notably, no selection bias was present with regard to the patients recruited for this study because SUH is the only hospital that provides care for the target population. Thus, the results might have external validation with regard to other Western populations. However, the results might not apply to perforated ulcer outcomes in regions where this disease has a different patient profile, such as Africa. Accordingly, to build on the results from this study, an international cohort of patients from various geographic regions should be sought. In addition, a unified agreement concerning data variables and inclusion is needed because these standards can differ across studies.

Globally, PPU is associated with a major surgical disease burden; however, randomised trials and prospective investigations are few and far between [37]. Additional international collaborations to increase the power of trials to generate more robust results should be pursued to improve care and eventually outcomes [38]. One of the predetermining factors for creating trials or comparing outcomes in PPU management is the possibility of allocating patients to risk based on agreed methods and consistent definitions. If the implementation of ANN is validated in larger and external series, this modelling might prove beneficial in terms of risk stratification or treatment allocation in a prospective setting. Notably, the current ANN model (AUC of 0.90) correctly identified 9 out of 10 patients at risk of dying within 30 days after PPU surgery. Furthermore, the long-standing issue of non-operative treatment and defining the best candidates for this treatment does not currently have acceptable prediction models [39]. Thus, focus should be placed on improving the prediction accuracy to generate reliable and robust models for future risk stratification and potential treatment allocation for patients with PPU.

Conflict of interest All authors declare that they have no conflicts.

Ethical standard The study was approved as a quality control assurance project according to the Regional Ethics Committee (REK Vest \# 2011/713).

Open Access This article is distributed under the terms of the Creative Commons Attribution License which permits any use, distribution, and reproduction in any medium, provided the original author(s) and the source are credited.

\section{References}

1. Stewart B, Khanduri P, McCord C, Ohene-Yeboah M, Uranues S, Vega Rivera F, Mock C. Global disease burden of conditions requiring emergency surgery. Br J Surg. 2014;101:e9-22.

2. Lau JY, Barkun A, Fan DM, Kuipers EJ, Yang YS, Chan FK. Challenges in the management of acute peptic ulcer bleeding. Lancet. 2013;381:2033-43.

3. Moller MH, Larsson HJ, Rosenstock S, Jorgensen H, Johnsen SP, Madsen AH, Adamsen S, Jensen AG, Zimmermann-Nielsen E, Thomsen RW. Quality-of-care initiative in patients treated surgically for perforated peptic ulcer. Br J Surg. 2013;100:543-52.

4. Ben-Ishay O, Bahouth $\mathrm{H}$, Kluger Y. Perforated peptic ulcer: determinants of outcome and mortality. J Emerg Trauma Shock. 2013;6:61.

5. Thorsen K, Glomsaker TB, von Meer A, Søreide K, Søreide JA. Trends in diagnosis and surgical management of patients with perforated peptic ulcer. J Gastrointest Surg. 2011;15:1329-35.

6. Hemmer PH, de Schipper JS, van Etten B, Pierie JP, Bonenkamp JJ, de Graaf PW, Karsten TM. Results of surgery for perforated gastroduodenal ulcers in a dutch population. Dig Surg. 2011;28:360-6.

7. Byrge N, Barton RG, Enniss TM, Nirula R. Laparoscopic versus open repair of perforated gastroduodenal ulcer: A national surgical quality improvement program analysis. Am J Surg. 2013;206:957-62 (discussion 962-953).

8. Møller MH, Adamsen S, Thomsen RW, Møller AM. Preoperative prognostic factors for mortality in peptic ulcer perforation: a systematic review. Scand J Gastroenterol. 2010;45:785-805. 
9. Thorsen K, Søreide JA, Søreide K. Scoring systems for outcome prediction in patients with perforated peptic ulcer. Scand $\mathbf{J}$ Trauma Resuscitation Emerg Med. 2013;21:25.

10. Boey J, Choi SK, Poon A, Alagaratnam TT. Risk stratification in perforated duodenal ulcers. A prospective validation of predictive factors. Ann Surg. 1987;205:22-6.

11. Thorsen K, Søreide JA, Søreide K. What is the best predictor of mortality in perforated peptic ulcer disease? A population-based, multivariable regression analysis including three clinical scoring systems. J Gastrointest Surg 2014. doi:10.1007/s11605-0142485-5.

12. Tepas JJ 3rd, Rimar JM, Hsiao AL, Nussbaum. Automated analysis of electronic medical record data reflects the pathophysiology of operative complications. Surgery. 2013;154:918-24 (discussion 924-916).

13. Stabile M, Cooper L. Review article: the evolving role of information technology in perioperative patient safety. Can J Anaesth. 2013;60:119-26.

14. Fogel SL, Baker CC. Effects of computerized decision support systems on blood glucose regulation in critically ill surgical patients. J Am Coll Surg. 2013;216:828-33 (discussion 833-825).

15. Cohen MJ. Use of models in identification and prediction of physiology in critically ill surgical patients. Br J Surg. 2012;99: 487-93.

16. Ansari D, Nilsson J, Andersson R, Regner S, Tingstedt B, Andersson B. Artificial neural networks predict survival from pancreatic cancer after radical surgery. Am J Surg. 2013;205:1-7.

17. Prabhudesai SG, Gould S, Rekhraj S, Tekkis PP, Glazer G, Ziprin P. Artificial neural networks: Useful aid in diagnosing acute appendicitis. World J Surg. 2008;32:305-9 (discussion 310-301).

18. Cucchetti A, Vivarelli M, Heaton ND, Phillips S, Piscaglia F, Bolondi L, La Barba G, Foxton MR, Rela M, O'Grady J, Pinna AD. Artificial neural network is superior to meld in predicting mortality of patients with end-stage liver disease. Gut. 2007;56: 253-8.

19. Selaru FM, Xu Y, Yin J, Zou T, Liu TC, Mori Y, Abraham JM, Sato F, Wang S, Twigg C, Olaru A, Shustova V, Leytin A, Hytiroglou P, Shibata D, Harpaz N, Meltzer SJ. Artificial neural networks distinguish among subtypes of neoplastic colorectal lesions. Gastroenterology. 2002;122:606-13.

20. von Elm E, Altman DG, Egger M, Pocock SJ, Gotzsche PC, Vandenbroucke JP, Initiative S. The strengthening the reporting of observational studies in epidemiology (strobe) statement: guidelines for reporting observational studies. Lancet. 2007;370: 1453-7.

21. Søreide K, Kørner H, Søreide JA. Diagnostic accuracy and receiver-operating characteristics curve analysis in surgical research and decision making. Ann Surg. 2011;253:27-34.

22. Boey J, Wong J, Ong GB. A prospective study of operative risk factors in perforated duodenal ulcers. Ann Surg. 1982;195:265-9.

23. Møller MH, Engebjerg MC, Adamsen S, Bendix J, Thomsen RW. The peptic ulcer perforation (pulp) score: a predictor of mortality following peptic ulcer perforation. A cohort study. Acta Anaesthesiol Scand. 2012;56:655-62.

24. Waljee AK, Higgins PD, Singal AG. A primer on predictive models. Clin Trans Gastroenterol. 2014;5:e44.

25. Jones N. Computer science: the learning machines. Nature. 2014;505:146-8.

26. Bierbrier R, Lo V, Wu RC. Evaluation of the accuracy of smartphone medical calculation apps. J Med Internet Res. 2014;16:e32.

27. Garg AX, Adhikari NK, McDonald H, Rosas-Arellano MP, Devereaux PJ, Beyene J, Sam J, Haynes RB. Effects of computerized clinical decision support systems on practitioner performance and patient outcomes: a systematic review. JAMA J Am Med Assoc. 2005;293:1223-38.

28. Glomsaker TB, Søreide K. Surgical training and working time restriction. Br J Surg. 2009;96:329-30.

29. Manning T, Sleator RD, Walsh P. Biologically inspired intelligent decision making: a commentary on the use of artificial neural networks in bioinformatics. Bioengineered. 2013;5(2):80-95.

30. Cleophas TJ, Cleophas TF. Artificial intelligence for diagnostic purposes: principles, procedures and limitations. Clin Chem Lab Med CCLM/FESCC. 2010;48:159-65.

31. Patel JL, Goyal RK. Applications of artificial neural networks in medical science. Curr Clin Pharmacol. 2007;2:217-26.

32. Grossi E, Mancini A, Buscema M. International experience on the use of artificial neural networks in gastroenterology. Dig Liver Dis. 2007;39:278-85.

33. Shi HY, Lee KT, Wang JJ, Sun DP, Lee HH, Chiu CC. Artificial neural network model for predicting 5-year mortality after surgery for hepatocellular carcinoma: a nationwide study. J Gastrointest Surg. 2012;16:2126-31.

34. Shi HY, Lee KT, Lee HH, Ho WH, Sun DP, Wang JJ, Chiu CC. Comparison of artificial neural network and logistic regression models for predicting in-hospital mortality after primary liver cancer surgery. PLoS ONE. 2012;7:e35781.

35. Rotondano G, Cipolletta L, Grossi E, Koch M, Intraligi M, Buscema M, Marmo R. Artificial neural networks accurately predict mortality in patients with nonvariceal upper gi bleeding. Gastrointest Endosc. 2011;73:218-26 (226 e211-212).

36. Andersson B, Andersson R, Ohlsson M, Nilsson J. Prediction of severe acute pancreatitis at admission to hospital using artificial neural networks. Pancreatology. 2011;11:328-35.

37. Søreide K, Thorsen K, Søreide JA. Strategies to improve the outcome of emergency surgery for perforated peptic ulcer. Br J Surg. 2014;101:e51-64.

38. Søreide K, Alderson D, Bergenfelz A, Beynon J, Connor S, Deckelbaum DL, Dejong CH, Earnshaw JJ, Kyamanywa P, Perez RO, Sakai Y, Winter DC. Strategies to improve clinical research in surgery through international collaboration. Lancet. 2013;382:1140-51.

39. Cao F, Li J, Li A, Fang Y, Wang YJ, Li F. Nonoperative management for perforated peptic ulcer: Who can benefit? Asian J Surg 2014. doi:10.1016/j.asjsur.2013.10.002. 